\title{
Professionalism in Medicine
}

\author{
Madawa Chandrathilake
}

During the recent past, professionalism emerged as an explicit competence or outcome of modern medical curricula (Harden, 1999; Frank \& Danoff, 2007; Zaini et al 2011; Tsugawa, 2009). The primary reason is that the general public in many countries repeatedly raised concerns on the professional conduct of doctors, compelling regulatory and professional bodies to take action. For example, isolated but significant incidents like the infamous case of Harold Shipman, a general practitioner who killed hundreds of patients under his care, triggered substantial reforms to the professional practice guidelines of the General Medical Council in the UK; 'professionalism' became a formal requirement to practice medicine (Jewell, 2005). The selfregulation of the profession by doctors themselves has been replaced with enhanced public scrutiny (Irvine, 2001). In USA, there were serious concerns about the way doctors manage conflict between self-interests and the interests of patients; altruism emerged as one of the corner stones of medical professionalism (American Board of Internal Medicine, ABIM: Project Professionalism Philadelphia, 2001). In addition, emerging evidence suggests that students or trainees, who demonstrated lapses of professionalism, are likely to face fitness to practice issues as future practitioners (Papadakis, 2004).

\section{Dynamics of professionalism}

Professionalism is not a static but a dynamic concept which has been changing throughout the history of allopathic medicine in response to societal changes and expectations (Sox, 2007). During the time of Hippocrates, doctors were similar to soothsayers, as the knowledge on disease processes was very limited.

Senior Lecturer in Medical Education,

Head of Medical Education Centre, Faculty of Medicine, University of Kelaniya, Sri Lanka
The concept of professionalism, therefore, was focused on the formation of the identity of medicine as a profession by adopting the basic elements of 'evidence-based approach to practice' (Sox, 2007). In the $19^{\text {th }}$ century, during which the industrial revolution was in its peak, 'professionalism' was focused on protecting patients from being mere victims of commercial and research interests of booming scientific and technological advancements (Sox, 2007). In the last few decades, with increasing demand for patient-centred medical practice, 'professionalism' has become a process of scrutinising the professional autonomy of doctors from the perspective of patients (Frank \& Danoff, 2007).

Therefore, professionalism has increasingly been recognised as a social contract between doctors and society (Cruess \& Cruess, 2000) Because of its nature, defining professionalism is challenging and it is further complicated by its sensitivity to cultural backgrounds (Chandratilake et al., 2012; Al-Eraky \& Chandratilake, 2012). What is defined as professionalism or lack of professionalism by the Western cultures may or may not make the same sense in Eastern cultures. Therefore, currently medical educationists and researchers attempt to 'understand' professionalism in relation to their own cultural and society context. However, there is general agreement that it encompasses a set of values, attitudes and behaviours. These attributes represent not only the relationship of doctors with patients, but their relationship with colleagues and other healthcare professionals in the team, and their behaviour in society (Chandratilake, 2010). The attributes of professionalism appear to range from personal qualities (e.g. honesty and integrity, managing conflicts of interest) to regulatory requirements (e.g., ethical conduct, accountability) to skills (e.g. effective communication, reflective practice, teamwork) (Hodges et al., 2011). 


\section{Fostering professionalism}

The nature of the concept naturally make us sceptic on the educability of professionalism. Can professionalism be taught, learned or be assessed? There is a growing body of evidence to suggest that it is an educable concept. Skills like communication, (Aspegren, 1999) reflection (Henderson \& Johnson, 2002) or teamwork (Aarnio et al., 2010) have been known as teachable constructs. Even certain personal qualities like honesty and integrity could be fostered (Bryan \& Babelay, 2009). However, it is important to understand that these diverse groups of attributes cannot be inculcated in our students using a single approach.

In any educational programme, there are three facets of the curriculum operating in parallel: formal curriculum, i.e. what is planned and documented; informal, i.e. what is not planned and documented but what is known to be happening in the educational settings; and hidden curriculum, i.e. what is unknowingly transmitted to learners through the institutional culture (Hafferty, 1998). It is not difficult to include skills components of professionalism in the formal curriculum. In fact, these have already been incorporated to many modern curricula. For example, most of us as clinicians take part in explicit teaching and assessment of communication skills. However, encompassing ethical values and conduct, and personal qualities within the limits of the formal curriculum alone would not be entirely successful. For example, we could teach students the correct technique of hand washing and the ethics behind doing it properly as a moral obligation towards patient safety, and assess these aspects in simulated environments (e.g. OSCE). However, in wards, clinics or theatres, hand washing may not take place in the same vein as taught to students. In rare situations, there may not be proper hand washing practice at all in certain settings. When students are exposed to clinical environments they face a dilemma between what is learned and what is practised, and often they succumb to the influence of the latter, i.e. informal or hidden aspects of curriculum. Therefore, the informal and hidden aspects of curricula play a greater role in fostering some aspects of professionalism
(Hafferty, 1998). This does not mean that the formal curriculum should not explicitly address these aspects. Rather, students should be aware of morality and moral conduct by providing knowledge, experiential learning opportunities, and reflection on practice or observations (Cruess \& Cruess, 2006). Most such attributes, however, are socially learned and are sustainable only in conducive institutional environments (Hafferty, 1998). Even at the early stages of medical education, teachers and clinicians become role models for students, and their behaviours, expressions and conduct become the sources and drivers of the informal and hidden curricula (Hafferty, 1998; Cruess et al., 2008). Our actions or inactions and commissions or omissions as clinicians ultimately influence the formation of professional identities of our students. Therefore, the professional culture of an institution plays a vital role in fostering professionalism among students and trainees. Professional culture can be explored through attitude surveys, student narratives, observations and critical incident analysis, and may be manipulated accordingly to achieve the desired educational outcomes. The primary goal of 'teaching' professionalism, however, should be to open up the informal and hidden components of the professionalism curriculum through student reflections and discussions, and to provide conducive environments and appropriate platforms for the purpose in the formal curriculum.

Medical educationists have taken several approaches to assessing professionalism. However, the lack of a universally agreed definition for professionalism has made assessment challenging (van Mook et al., 2009). The assessment of students' or trainees' awareness of professional expectations and requirements, and demonstration of this awareness appears to be the most basic approach. The commonly used assessment methods include written tests such as scenario-based multiple choice questions, Extended Matching Items, and OSCEs (Wilkinson et al., 2009). Although this step is important, students can 'fake' professional attitudes and behaviours in such assessments, i.e. demonstrating professionalism rather than becoming professionals (Rees \& Knight, 2007). 
Therefore, the assessment of professionalism should essentially be extended beyond the assessment of the cognitive base. The assessment of reflection in and on practice on professionalism issues (Wilkinson et al., 2009) may be a step higher than the assessment of the cognitive base. However, it should most appropriately be assessed in educational or work-place environments using observations and multisource feedback (MSF) (Wilkinson et al., 2009). Observation of students / trainees for their professional approach to day-to-day clinical practice is an essential component of the assessment process. However, observations warrant considerable amount of staff time which may be a limiting factor of using this method in resource-limited clinical environments. In addition, collated perceptions of various members of the healthcare delivery team (e.g. peers and nurses) or different stakeholder groups (e.g. managers, patients, etc. in addition to the team members) would contribute substantially to understand professionalism of individuals. The role of assessing 'doctors' by other healthcare professionals or patients in situations such as MSF, however, may not be acceptable in certain cultures. Although it is an issue of professionalism, this attitude may threaten the utility of such assessments. Observations and MSF should be used repeatedly and in multiple settings to obtain credible insight into professional behaviour of students or trainees (Wilkinson et al., 2009). Unlike clinical competence or incompetence, determining professional or unprofessional behaviour may not be straight forward or 'black and white'; assessors may feel 'uncomfortable' of failing students or trainees solely on the grounds of professionalism and such decisions may not be entirely 'acceptable' to assessors (Rees \& Knight, 2009). This issue is further complicated by the fact that most professional lapses are less 'dramatic' but vital for patient safety, and there may not be consensus on how to respond to them (Roff et al., 2011). Therefore, although summative assessments are necessary to ensure patient safety and professional accountability, professionalism should be assessed primarily for providing feedback and facilitating the development of right professional persona, i.e. assessing formatively (Hodges et al., 2011). The main emphasis of professionalism assessment, therefore, should be assessment for learning and such assessments should be carried out with a sound mutual understanding between the assessor and assessee on the constructive nature of the process.

In summary, professionalism should be understood in the backdrop of culture and context. It is learned by students primarily from role models. The professional culture of institutions also plays a major role in fostering professionalism. It should be assessed mainly to facilitate further learning, and multisource feedback and observations are the most useful methods of assessment. Amidst the changeling nature, being explicit on the expected professional behaviour and taking steps to inculcate its elements in students and trainees has become important and essential in today's context.

\section{References}

Aarnio, M., Nieminen, J., Pyorala, E. \& LindblomYlanne, S. (2010) Motivating medical students to learn teamwork skills, Medical Teacher, 32, 4, pp.199-204.

American Board of Internal Medicine, Project Professionalism Philadelphia (2001) American Board of Internal Medicine, pp.1 - 31.

Al-Eraky, M.M. \& Chandratilake, M. (2012) How medical professionalism is conceptualised in Arabian context: A validation study, Medical Teacher, 34, pp.90-95.

Aspegren, K. (1999) BEME Guide No. 2: Teaching and learning communication skills in medicine a review with quality grading of articles, Medical Teacher, 21, 6, pp.563-570.

Bryan, C.S. \& Babelay, A.M. (2009) Building character: a model for reflective practice, Academic Medicine 84, 9, pp.1283-1288.

Chandratilake, M., McAleer, S. \& Gibson, J. (2012) Cultural similarities and differences in medical professionalism: a multi-region study, Medical Education, 46, 3, pp.257-266.

Chandratilake, M., McAleer, S., Gibson, J. \& Roff, S. (2010) Medical professionalism: what does the public think? Clinical Medicine, 10, 4, pp. 364-369.

Cruess, R.L. \& Cruess, S. (2006) Teaching professionalism: general principles, Medical Teacher, 28, 3, pp.205-208.

Cruess, S.R. \& Cruess, R.L. (2000) Professionalism: a contract between medicine and society, Journal of Ayub Medical College,162, 5, pp.668 - 669. 
Cruess, S.R., Cruess, R.L. \& Steinert, Y. (2008) Teaching rounds - Role modelling - making the most of a powerful teaching strategy, British Medical Journal, 336, 7646, pp.718-721.

Frank, J.R. \& Danoff, D. (2007) The CanMEDS initiative: implementing an outcomes-based framework of physician competencies, Medical Teacher, 29, 7, pp.642-647.

Hafferty, F.W. (1998) Beyond curriculum reform:confronting medicine's hidden curriculum, Academic Medicine 73, pp.403 - 407.

Harden, R.M. (1999) AMEE Guide No. 14: Outcome-based education: Part 1-An introduction to outcome-based education, Medical Teacher, 21, 1, pp.7-14.

Henderson, P. \& Johnson, M.H. (2002) An innovative approach to developing the reflective skills of medical students, BMC medical education, 2, p.4.

Hodges, B.D., Ginsburg, S., Cruess, R., Cruess, S., Delport, R. \& Hafferty, F. (2011) Assessment of professionalism: recommendations from the Ottawa 2010 Conference, Medical Teacher, 33, 5, pp.354-63.

Irvine, D. (2001) Doctors in the UK: their new professionalism and its regulatory framework, Lancet, 358, 9295, pp.1807-1810.

Jewell, D. (2005) After shipman: reforming the GMC - again, British Journal of General Practice, 55, 511, pp.83-84

Papadakis, M.A., Hodgson, C.S., Teherani, A. \& Kohatsu, N.D. (2004) Unprofessional behavior in medical school is associated with subsequent disciplinary action by a state medical board, Academic Medicine, 79, 3, pp.244-249.
Rees, C.E. \& Knight, L.V. (2007) The trouble with assessing students' professionalism: theoretical insights from sociocognitive psychology, Academic Medicine, 82, 1, pp.46-50.

Rees, C.E., Knight, L.V. \& Cleland, J.A. (2009) Medical educators' metaphoric talk about their assessment relationships with students: 'you don't want to sort of be the one who sticks the knife in them', Assessment and Evaluation in Higher Education, 34, 4, pp.455-467.

Roff, S., Chandratilake, M., McAleer, S. \& Gibson, J. (2011) Preliminary benchmarking of appropriate sanctions for lapses in undergraduate professionalism in the health professions, Medical Teacher, 33, 3, pp.234-238.

Sox, C.H. (2007) The ethical foundations of professionalism; a sociologic history, Chest, 131, 5, pp.1532-1540.

Tsugawa, Y., Tokuda, Y., Ohbu, S., Okubo, T., Cruess, R. \& Cruess, S. (2009) Professionalism Mini- Evaluation Exercise for medical residents in Japan: a pilot study, Medical Education, 43, 10, 968-978.

Van Mook, W., van Luijk, S., O'Sullivan, H., Wass, V., Schuwirth, L. \& van der Vleuten, C. (2009) General considerations regarding assessment of professional behaviour, European Journal of Internal Medicine, 20, pp.90-95.

Wilkinson, T.J., Wade, W.B. \& Knock, L.D. (2009) A blueprint to assess professionalism: results of a systematic review, Academic Medicine, 84, 5, pp.551-558.

Zaini, R.G., Bin Abdulrahman, K.A., Al-Khotani, A.A, Al-Hayani, A.M, Al-Alwan, I.A. \& Jastaniah, S.D.(2011) Saudi Meds: a competence specification for Saudi medical graduates, Medical Teacher, 3, 7, pp.582-584. 